\title{
Exploring the Neuroprotective Effects of Mechanosensitive Channel Blocker, GsMTX4 in Intracerebral Hemorrhage Model in Rats
}

\author{
Wenyan Liu', Guiyu Zhang', Jin Wang', Jing Liu', Yu Yuan', Jun Hong1, Yi Ding2,* \\ 'Department of Neurosurgery, Tangshan Gongren Hospital, Tangshan, Hebei, CHINA. \\ ${ }^{2}$ Surgical Intensive Care Unit, Chongqing Jiangjin Central Hospital, Chongqing, CHINA.
}

\begin{abstract}
Aim: The current study explored the usefulness of a mechanosensitive channel blocker, GsMTX4 in intracerebral hemorrhage (ICH)-associated deleterious effects along with the possible mechanisms. Materials and Methods: Type VII collagenase was administered in the right basal ganglia using stereotactic apparatus to induce $\mathrm{ICH}$ in rats. The $\mathrm{ICH}-$ associated injury was assessed using corner turn and forelimb placement tests (behavioral test); Evans blue extravasation test (blood-brain barrier damage), brain edema, apoptotic markers (caspase- 3 and Bcl-2 levels). The levels of TNF- $\alpha$ (neuroinflammation), reactive oxygen species, and Brain-derived neurotrophic factor (BDNF) were also measured in $\mathrm{ICH}$-subjected rats. GsMTx4, as a mechanosensitive channel blocker, and ANA-12, as a selective BDNF receptor blocker were employed as pharmacological agents. Results: Administration of GsMTx4 attenuated $\mathrm{ICH}$-associated behavioral changes, preserved blood-brain barrier, prevented brain edema, and decreased apoptosis. GsMTx4 also decreased neuroinflammation and oxidative stress, while it increased the levels of BDNF. Moreover, administration of ANA-12 attenuated the neuroprotective effects of GsMTx4 suggesting that BDNF may be important in inducing neuroprotective effects of GsMTx4. Conclusion: GsMTx4 exerts neuroprotective effects in intracerebral hemorrhageassociated deleterious effects, which may be possibly due to an increase in BDNF levels along with a decrease in neuroinflammation and oxidative stress.
\end{abstract}

Key words: Neuroinflammation, Oxidative stress, Intracerebral hemorrhage, Mechanosensitive channels, BDNF.

\section{INTRODUCTION}

Stroke is a neurological emergency, which is characterized by sudden focal neurologic deficit lasting for at least $24 \mathrm{~h}$ and it mainly arises due to problems related to blood flow. Ischemic stroke, characterized by a decrease in blood supply to the brain, is the more prevalent form of stroke. However, the prognosis is much better in patients with cerebral ischemia in comparison to the hemorrhagic form of stroke. ${ }^{1}$ The characteristic feature of intracerebral hemorrhage $(\mathrm{ICH})$ involves the rupture of cerebral blood vessels, which leads to very high mortality, around $40 \%$ in strokeaffected population. ${ }^{2}$ The major focus on the management of ICH has been on effectivelyand rapidly lowering blood pressure, controlling the hemostasis by transfusing platelets and clotting complexes, and employment of invasive techniques to reduce clot volume. ${ }^{3,4}$ In recent years, there have been studies exploring new neuroprotective interventions to limit neuronal damage associated with ICH. ${ }^{5,6}$

Recent studies have shown the neuroprotective actions of mechanosensitive ion channel blockers. There have been studies showing the blood-brain barrier protecting actions of a pharmacological inhibitor of transient receptor potential vanilloid 4 (TRPV4), a
Submission Date: 06-10-2020; Revision Date: 25-01-2021; Accepted Date: 12-04-2021

DOI: 10.5530/ijper.55.3.148 Correspondence:

Dr. Yi Ding

Surgical Intensive Care Unit, Chongqing Jiangjin Central Hospital, Chongqing-402260, CHINA.

Phone no: +86-15112009654 Email id: dingyi321654@ sina.com

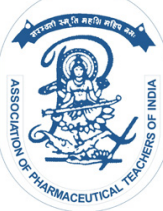

www.ijper.org 
mechanosensitive channel, in $\mathrm{ICH}^{7}$ Gadolinium, a non-selective mechanosensitive channel blocker, has also been shown to produce neuroprotection in intracerebralhemorrhage. ${ }^{8}$ Piezochannels are therelatively new members of mechanically activated ion channels. ${ }^{9}$ These channels are widely distributed in the body including the lungs, gastrointestinal tract, bladder, and skin. ${ }^{10}$ Moreover, these channels are also abundantly present in the central nervous system. ${ }^{11,12}$ GsMTx4 is a 35 amino acid peptide derived from Grammostola spatulata and it has been found to selectively inhibit mechano-gated piezo channels. ${ }^{913}$ Apart from the Piezo 1 and 2 channels, GsMTx4 has also been shown to block mechanosensitive channels, such as TRPC1 and TRPC6. ${ }^{14}$ Studies have shown the beneficial effects of GsMTx4 in different conditions, including ischemiareperfusion-induced neuronal injury; ${ }^{15}$ demyelination in the central nervous system ${ }^{16}$ and muscular dystrophy ${ }^{17}$ etc. However, the neuroprotective role of GsMTx4 in ICH models still needs to be identified. Accordingly, the current study was aimed to explore the therapeutic potential of a mechanosensitive channel blocker, GsMTX4 in ICH-associated deleterious effects along with the possible mechanisms.

\section{MATERIALS AND METHODS}

\section{Animals and drugs}

Male Wistar albino rats (200-250 g) were obtained from Nanjing Junke Bioengineering Co, Ltd, Nanjing, China. The animals were kept in the animal house in the standardized laboratory conditions $\left(25 \pm 2^{\circ} \mathrm{C}, 50-60\right.$ $\%$ relative humidity, $12 \mathrm{~h}$ light/12 dark cycle). In total, 105 animals were used for this study. Animal Ethical Committee of Tangshan Gongren Hospital, China with Ethic Number: TGH0924 approved the experimental protocol. The ELISA kits for the estimation of TNF- $\alpha$ (ab46070), BDNF (ab213899) and caspase-3 (ab39401) were obtained from abcam, Cambridge, USA. The ELISA kit for the estimation of Bcl-2 (E-EL-R0096) was obtained from Elabscience Houston, TX 77079, USA. The drugs employed in this study i.e. GsMTx4 and ANA-12 along with collagenase VII, Evans blue, 2',7'-dichlorofluorescin diacetate (DCFH-DA), isoflurane, ketamine, xylazine and acepromazine were procured from Sigma-Aldrich, USA. The doses of GsMTx $4^{18}$ and ANA- $12^{19}$ were chosen based on earlier reports.

\section{ICH Model}

The animals were anesthetized using injecting ketamine (50 $\mathrm{mg} / \mathrm{kg})$, xylazine $(5 \mathrm{mg} / \mathrm{kg}$ ) and acepromazine $(1 \mathrm{mg} / \mathrm{kg})$ intraperitonially. In anesthetized rats, type VII collagenase ( $0.5 \mathrm{U}$ in $2 \mu \mathrm{L}$ saline/rat) was injected into the brain at a rate of $0.4 \mu \mathrm{L} / \mathrm{min}$ in a stereotactic frame. Briefly, a hole of $1 \mathrm{~mm}$ was made into the skull to insert the 26-gauge needle in the right basal ganglia. The stereotaxic coordinates were $0.2 \mathrm{~mm}$ anterior, $3 \mathrm{~mm}$ right lateral to the bregma, and $6 \mathrm{~mm}$ ventral to the skull. In the sham group, the same procedure was followed except for infusion of normal saline instead of collagenase VII. ${ }^{20}$

\section{Behavioral Testing}

After $24 \mathrm{~h}$ of ICH, behavioral testing was done, which included the followings:

\section{Corner turn test}

In this behavioral test, the rats were kept at a $30^{\circ}$ corner and these were allowed to make 10 turns in total (either right or left), with a time of $15 \mathrm{~s}$ between each turn. Amongst these, the total number of right turns made by the rats was recorded. Since there is an injury to the right side of the brain, therefore, there is more tendency to make a turn on the right side (ipsiversive) ( $n=15$ rats/group). The results of the corner turn test were expressed as percentage. ${ }^{20-22}$

\section{Forelimb Placing Test}

It is a vibrissae-elicited forelimb placing test (paw whisker test) and the damage to the motor system elicits impairment in paw placing. It is a very useful test to assess the development of asymmetry in the brain regions of the sensorimotor cortex and striatum. In this test, the rat was held in such a way that its four limbs were hanging freely. The testing of the forelimb was induced by brushing the vibrissae of the rat on the corner edge of the cornerstone. The injured rat quickly placed the forelimb on the cornerstone in response to the brushing of vibrissae on the corner edge, while there was impairment in the forelimb placing on the top of the table in injured rats. Ten trials were done on each side of the rat and the number of successful placements (placing the forelimb on the table) was recorded and expressed in percentage of total trials ( $n=15$ rats $/$ group). ${ }^{20-22}$

\section{Evans blue extravasation assay}

This test was employed to check the integrity of the blood-brain barrier after $24 \mathrm{~h}$ of ICH. In this test, $2 \%$ Evans blue dye $(2 \mathrm{ml} / \mathrm{kg})$ was injected into a lateral tail vein. After $2 \mathrm{~h}$, animals were sacrificed by allowing rats to inhale vapors of $8 \%$ isoflurane in a closed chamber. Thereafter, the brains were removed. The weights of these brains were noted and thereafter, treatment with formamide was done for $24 \mathrm{~h}$ at $37^{\circ} \mathrm{C}$. Afterward, the brain samples were subjected to centrifugation at 2,500 $\mathrm{g}$ 
for 15 min to obtain clear supernatant, whose absorbance was recorded at $632 \mathrm{~nm}^{20,21}$ ( $n=5$ rats/group).

\section{Brain Edema Test}

The brain edema was measured by determining the amount of water in the brain (after sacrificing the rats using $8 \%$ isoflurane). For this test, the brain was divided into two halves. The right half of the brain was weighed as the wet weight. Then, the brain portion was dried at $100^{\circ} \mathrm{C}$ for $24 \mathrm{~h}$ and weighed again as the dry weight. The brain water content was calculated of right half as a percentage ( $n=5$ rats/group). ${ }^{20,22}$

\section{Biochemical Analysis in brain homogenates}

The brains were removed after sacrificing the rats (using $5 \%$ isoflurane) and then homogenized in the phosphate buffer solution, $\mathrm{pH}=7.4$. The brain homogenate was centrifuged at $4000 \times \mathrm{g}$ for $15 \mathrm{~min}$ and the clear supernatant obtained was used to measure biochemical parameters ( $n=5$ rats/group). The supernatants were stored at $-70^{\circ} \mathrm{C}$ in a deep freezer till biochemical testing was performed.

\section{Apoptosis Markers}

The levels of caspase- 3 and Bcl-2 as markers of apoptosis were measured using commercially available ELISA kits. The values were expressed in terms of $\mathrm{ng} / \mathrm{mg}$ of protein.

\section{TNF- $\alpha$, BDNF and reactive oxygen species (ROS) levels}

The quantitative analysis of TNF- $\alpha$ and BDNF was performed using ELISA kits, and instructions to conduct analysis were followed as described in these commercial kits. The measurement of ROS levels was done by the treatment of brain homogenate with 2',7'-dichlorofluorescin diacetate (DCFH-DA) for 30 min at $37^{\circ} \mathrm{C}$. The intensity of fluorescence was checked at $484 \mathrm{~nm}$ (excitation splitter) and $530 \mathrm{~nm}$ (emission splitter), respectively. ${ }^{20-23}$

\section{Study Design}

The study included seven groups and each group comprised of 15 animals. The study design as explained below (Figure 1):

(i) Group I (Normal control): Animals were not subjected to any treatment and all tests were performed

(ii) Group II (Sham control): Animals were administered normal saline stereotactically and after $24 \mathrm{~h}$, all tests were performed

(iii) Group III (ICH control): Animals were administered collagenase VII in the brain and after $24 \mathrm{~h}$, all tests were performed

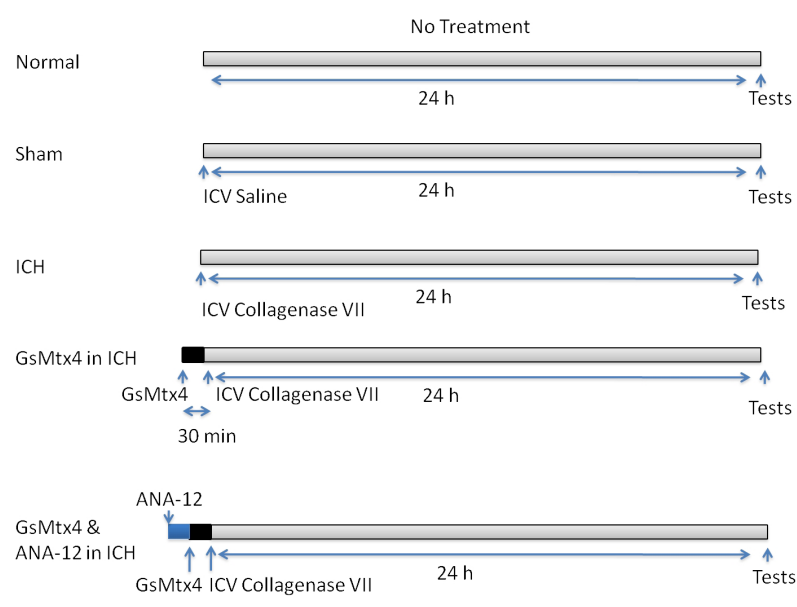

Figure 1: Diagrammatic representation of experimental design.

(iv) Group IV [GsMTx4 (5 mg/kg) in ICH]: GsMTx4 (5 $\mathrm{mg} / \mathrm{kg}$ i.v.) was administered $30 \mathrm{~min}$ before subjecting rats to $\mathrm{ICH}$ and after $24 \mathrm{~h}$ of $\mathrm{ICH}$, all tests were performed

(v) Group V [GsMTx4 (10 mg/kg) in ICH]: GsMTx4 (10 mg/kg i.v.) was administered $30 \mathrm{~min}$ before subjecting rats to ICH and after $24 \mathrm{~h}$ of $\mathrm{ICH}$, all tests were performed

(vi) Group VI [GsMTx4 (10 mg/kg) and ANA-12 (0.25 $\mathrm{mg} / \mathrm{kg})$ ]: ANA-12 (0.25 mg/kg i.p.) was administrated $1 \mathrm{~h}$ before, while GsMTx4 (10 $\mathrm{mg} / \mathrm{kg}$ i.v.) was administered $30 \mathrm{~min}$ before subjecting rats to ICH. After $24 \mathrm{~h}$ of ICH, all tests were performed

(vii) Group VII [GsMTx4 (10 mg/kg) and ANA-12 (0.50 $\mathrm{mg} / \mathrm{kg})$ ]: ANA-12 (0.5 mg/kg i.p.) was administrated $1 \mathrm{~h}$ before, while GsMTx4 (10 mg/kg i.v.) was administered 30 min before subjecting rats to ICH. After $24 \mathrm{~h}$ of ICH, all tests were performed.

\section{Statistical Analysis}

The GraphPad Prism 7 was employed for statistical analysis. The results were reported in the form of mean $\pm S D$. The comparison of experimental data was done using One Way ANOVA. This statistical test was followed by Tukey's multiple range test. The value of $p<0.5$ was considered to be statistically significant.

\section{RESULTS}

Development of behavioral abnormalities, brain edema, and disruption of the blood-brain barrier in ICH-subjected rats.

In response to collagenase VII administration in the right portion of the brain, there was a significant development of behavioral abnormalities assessed in 

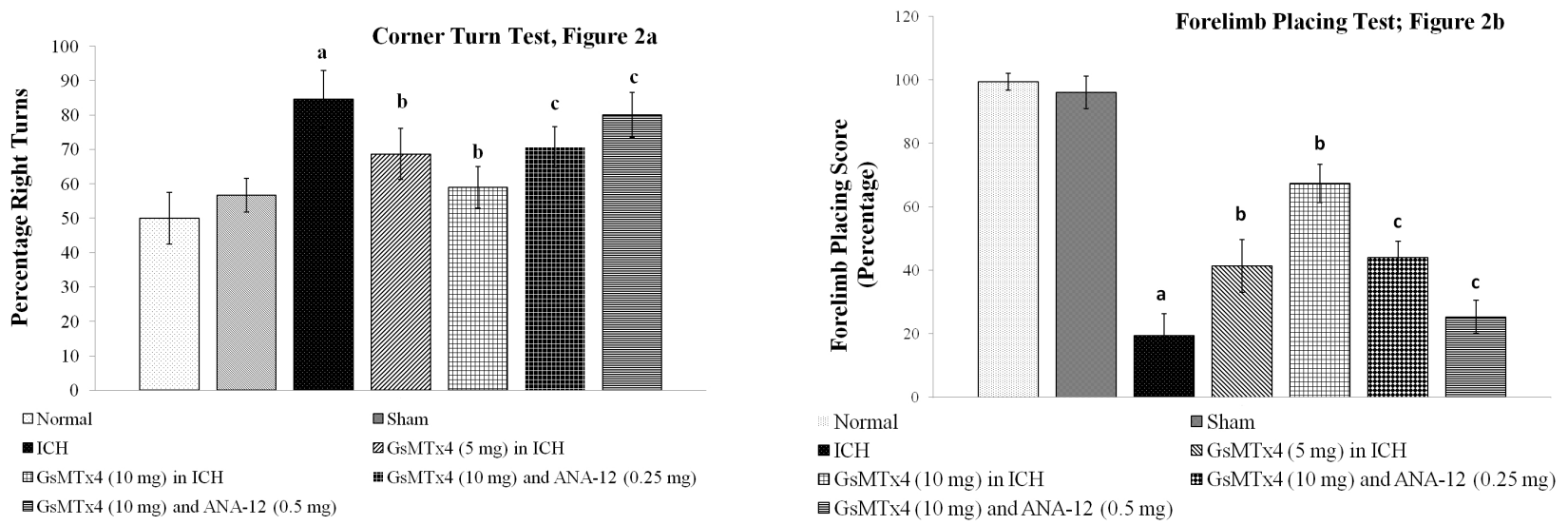

Figure 2: Effect of different treatments on corner turn test (2a) and forelimb placement test (2b). $a=p<0.05$ vs. sham; $\mathrm{b}=p<0.05$ vs. ICH; $\mathrm{c}=p<0.05$ vs. GsMTx4 $(10 \mathrm{mg} / \mathrm{kg})$ in ICH.
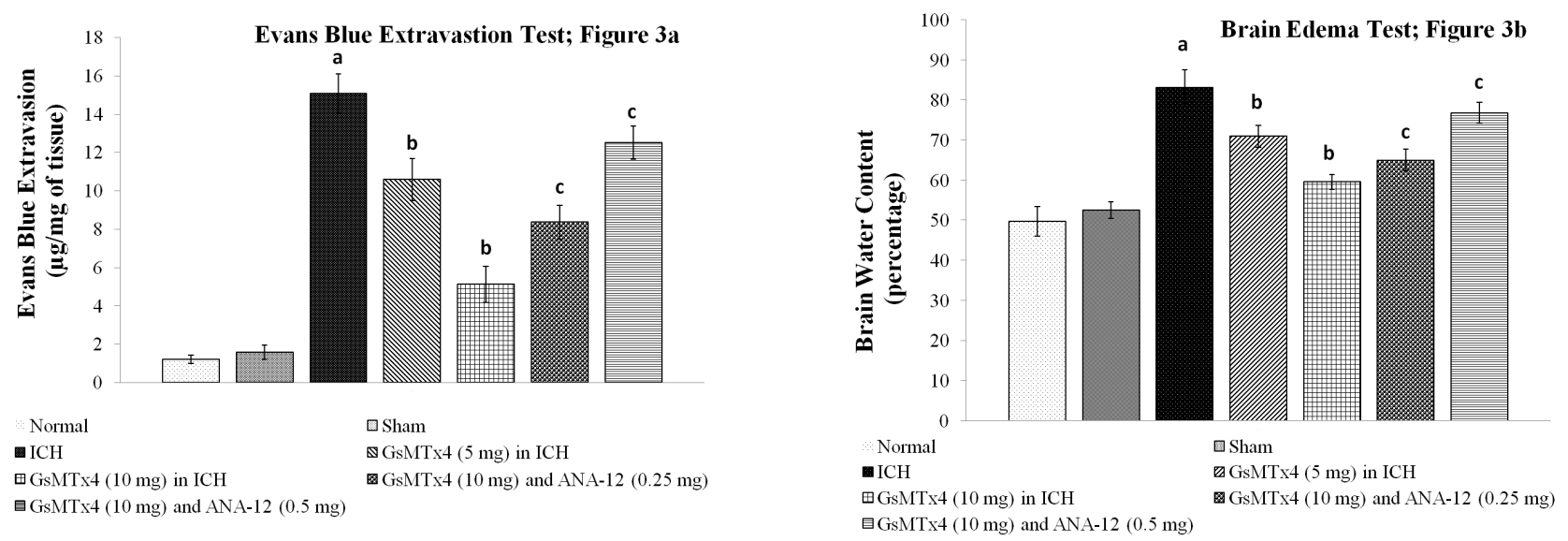

Figure 3: Effect of different treatments on Evans blue extravasation test (3a) and brain edema test (3b). $a=p<0.05$ vs. sham; $\mathrm{b}=p<0.05$ vs. ICH; $\mathrm{C}=p<0.05$ vs. GsMTx4 $(10 \mathrm{mg} / \mathrm{kg})$ in ICH.

the corner turn test and forelimb placing test. There was a marked rise in the number of right turns in the $\mathrm{ICH}$ model in the corner turn test (Figure 2a) and a decrease in the percentage of forelimb placing (Figure $2 \mathrm{~b}$ ) in comparison to sham and normal control. Moreover in collagenase VII administered rats, there was a significant increase in the absorbance in the brain homogenate following IV injection of Evan blue through tail vein, suggesting the leakage of blood-brain barrier. Along with the significant Evans blue extravasation (Figure 3a), there was an increase in the brain water content (Figure 3b) in ICH-subjected rats suggesting the significant disruption of the blood-brain barrier and brain edema.

\section{Biochemical alterations in the brains of $\mathrm{ICH}-$ subjected rats}

In ICH-subjected rats, a marked alteration in the apoptotic markers including an increase in caspase- 3 (proapoptotic) (Figure 4a) and a decrease in Bcl-2 (antiapoptotic) (Figure 4b) was observed in the brain homogenates in comparison to sham and normal control. It suggests a significant increase in the apoptotic cell injury in the ICH model. A marked rise in the TNF- $\alpha$ (Figure 5a) and a decrease in the BDNF levels (Figure 5b) were also observed in the brain homogenates of the ICH model. Moreover, there was also a rise in the levels of reactive oxygen species (Figure 5c) in rat brains, suggesting the increase in oxidative stress in response to ICH.

\section{GsMTx4 attenuated behavioral and other alterations in $\mathrm{ICH}-$ subjected rats}

Administration of GsMTx4 (5 and $10 \mathrm{mg} / \mathrm{kg}$ ) significantly attenuated $\mathrm{ICH}$-induced alterations in behavior (Figure 2), injury to blood-brain barrier (Figure $3 a$ ), increase in brain edema (Figure 3b), development of apoptotic cell injury (Figure 4), increase in TNF- $\alpha$ (Figure 5a) and reactive oxygen species (Figure 5c). Moreover, GsMTx4 also restored the levels of BDNF (Figure 5b) in ICH-subjected rats in a significant manner. 

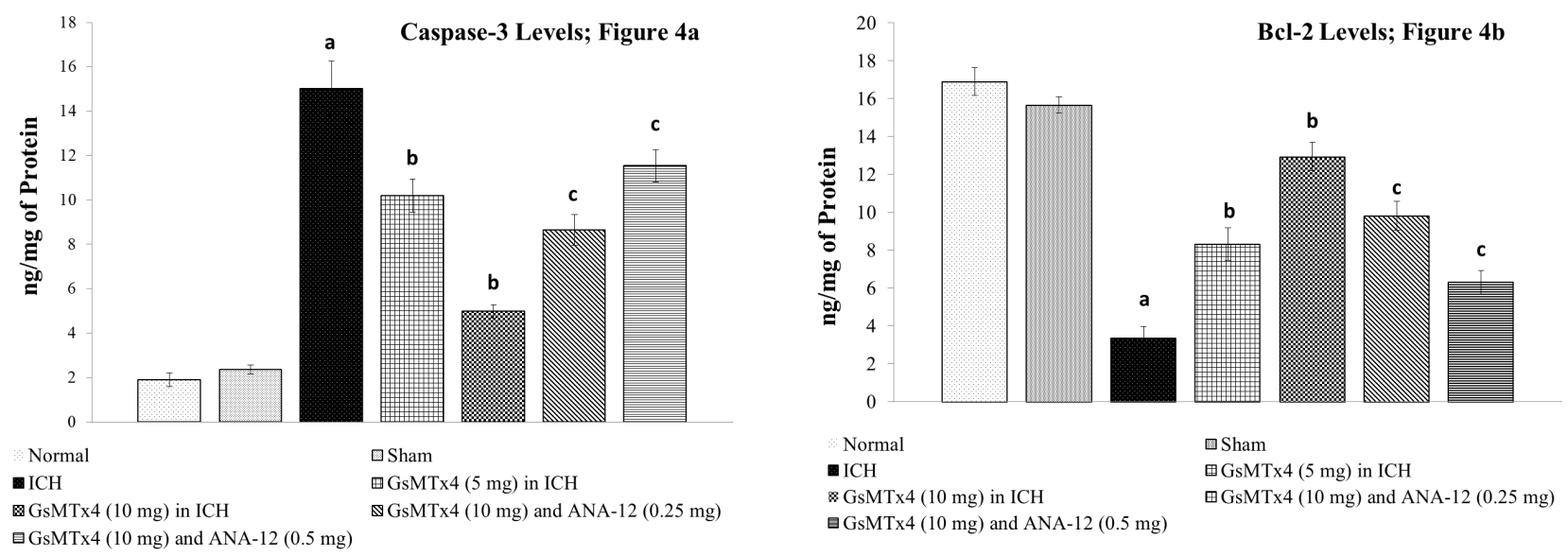

Figure 4: Effect of different treatments on apoptotic markers, caspase-3 (4a) and Bcl-2 (4b). $\mathrm{a}=p<0.05 \mathrm{vs.} \mathrm{sham;} \mathrm{b}=p<0.05$ vs. ICH; $\mathrm{C}=\boldsymbol{p}<0.05$ vs. GsMTx4 $(10 \mathrm{mg} / \mathrm{kg})$ in ICH.
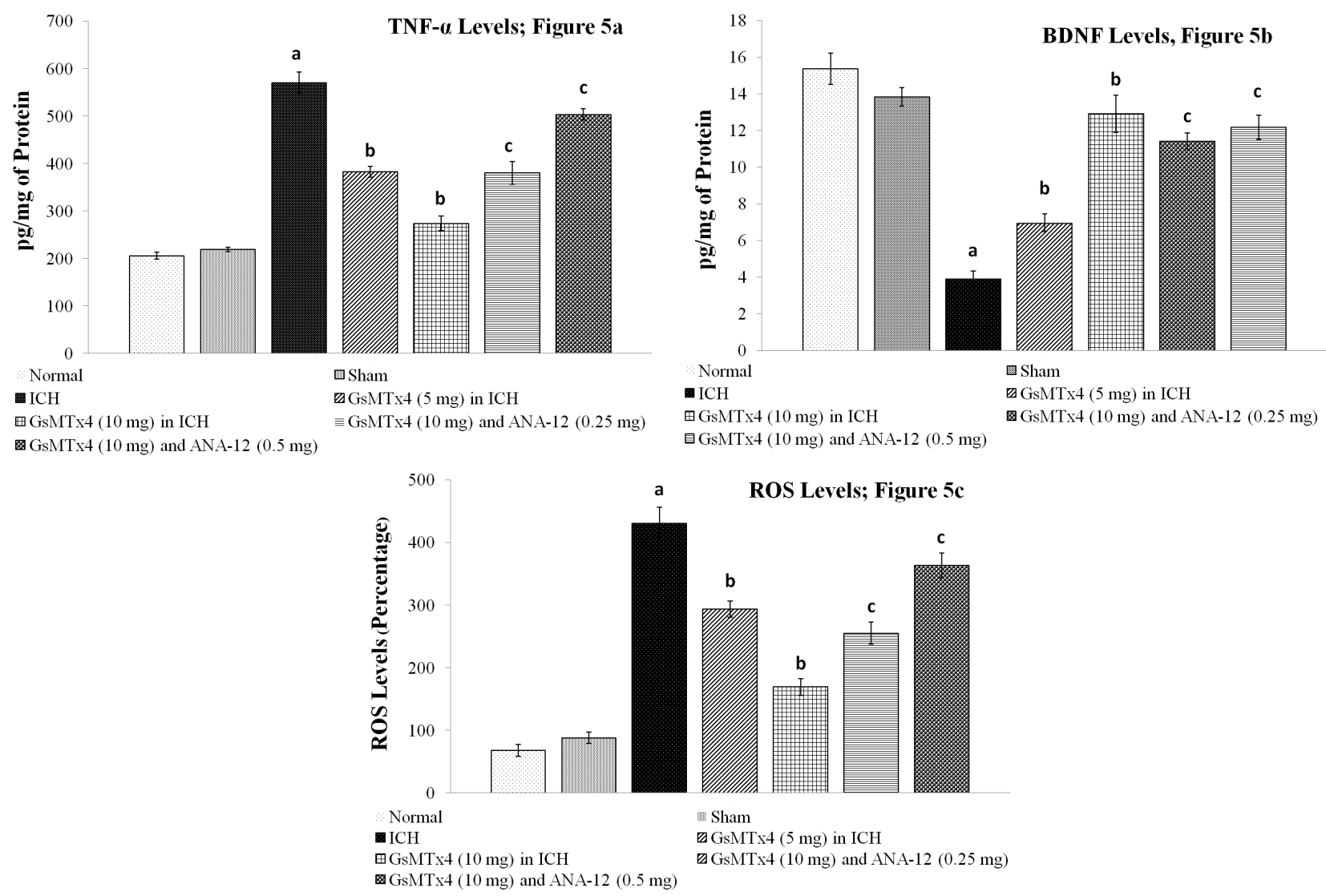

Figure 5: Effect of different treatments on TNF- $\alpha$ (5a), BDNF (5b) and reactive oxygen species (5c). a = $p<0.05$ vs. sham; $\mathrm{b}=p<0.05$ vs. ICH; $\mathrm{c}=p<0.05$ vs. GsMTx4 $(10 \mathrm{mg} / \mathrm{kg})$ in ICH.

\section{ANA-12 abolished the neuroprotective actions of GsMTx4 in ICH-subjected rats}

Treatment with ANA-12 (0.25 and $0.5 \mathrm{mg} / \mathrm{kg}$ ) abolished the neuroprotective actions of GsMTx4 in ICH-subjected rats. GsMTx4-mediated improvement in behavior, decrease in blood-brain injury, brain edema and apoptosis was abolished in ANA-12-treated rats. Moreover, the effects of GsMTx4 on TNF- $\alpha$ and oxidative stress were also abolished in ANA-12-treated rats. However, ANA12 did not modulate the levels of BDNF in GsMTx4treated rats. 


\section{DISCUSSION}

In the present study, injection of collagenase VII in the right portion of basal ganglia of rats produced the features of ICH including the increase in the tendency for right turns (corner turn test), decrease in forelimb placing (forelimb placing test), damage to the blood-brain barrier (Evans blue extravasation) and development of brain edema (brain water content test). Moreover, there was also an increase in apoptotic cell injury as assessed by a significant increase in the levels of proapoptotic (caspase-3) and a decrease in the levels of antiapoptotic $(\mathrm{Bcl}-2)$ in these rats. The results of this study are supported by the results of the earlier studies. ${ }^{20,22}$

In this study, administration of GsMTx4 produced neuroprotection against ICH-induced brain injury in a dose-dependent manner. In GsMTx4-treated rats, there was a significant improvement in the behavior of animals, a decrease in brain edema, blood barrier damage and markers of apoptosis. As a mechanosensitive channel blocker, GsMTx4 inhibits Piezo 1 and Piezo 2 channels ${ }^{9}$ along with TRPC1 and TRPC6. ${ }^{14}$ Piezo 1 channels are activated by mechanical pressure and these are widespread in the different parts of the body. ${ }^{24,25}$ Moreover, these are also abundantly present in the neurons of the brain. ${ }^{12,26}$ Studies have shown the contribution of Piezo 1 channels in the pathology of different diseases. ${ }^{11,25,27}$ Moreover, GsMTx4-sensitive other mechanosensitive channels including TRPC1 ${ }^{28}$ and TRPC $6^{29}$ are also present in the brain. Apart from it, GsMTx4 has been shown to attenuate ischemiareperfusioninjury toheart ${ }^{19}$ brain $;{ }^{15}$ preventdemyelination in the central nervous system, ${ }^{16}$ muscular dystrophy. ${ }^{17}$ It is the first report describing the effectiveness of GsMTx4 in producing beneficial effects in rats mimicking intracerebral hemorrhage.

In this study, ICH led to a rise in the levels of TNF- $\alpha$ (neuroinflammation) and reactive oxygen species (oxidative stress) suggesting that an increase in oxidative stress and neuroinflammation may be critical in inducing deleterious changes observed in $\mathrm{ICH}$-subjected rats. There have been studies showing that neuroinflammation and oxidative stress may be critical in the pathogenesis of intracerebral hemorrhage-induced deleterious effects. ${ }^{30,31}$ However, administration of GsMTx4 mitigated ICHassociated increase in neuroinflammation and oxidative stress. Previous studies have suggested the potential of GsMTx4 in decreasing inflammation and oxidative stress. $^{32,33}$ Accordingly, it may be suggested that the neuroprotective effects of GsMTx 4 in the intracerebral hemorrhage may be due to a decrease in inflammation and oxidative stress.
Apart from these alterations, the significant role of BDNF was also identified in the ICH model. There was a marked decrease in the BDNF levels in the brains of ICH-subjected rats. BDNF is a neurotrophic factor and its beneficial role in intracerebral hemorrhage has been well documented. ${ }^{34-36}$ Moreover, the observed neuroprotective effects of GsMTx4 in this study were associated with the increase in the brain BDNF levels, which suggests that GsMTx4-mediated beneficial effects in the ICH model may be secondary to arise in BDNF levels. The relationship between the inhibition of mechanosensitive channels with GsMTx4 and an increase in the BDNF levels is an interesting and needs further experimental studies. There have been previous studies showing that the inhibition of mechanosensitive channels by GsMTx4 stimulates the neurite outgrowth in the Xenopus spinal cord ${ }^{37}$ and PC12 cells. ${ }^{38}$ In these studies, GsMTx4 was shown to produce synergistic effects with the nerve growth factor (NGF) in promoting neuritic growth. Jacques-Fricke et al. proposed that inhibition of $\mathrm{Ca}^{2+}$ influx in the presence of GsMTx4 contributes in accelerating the neurite growth extension. ${ }^{37}$ However, more studies are required to explore the mechanisms involved in GsMTx4-mediated increase in the levels of BDNF. The role of BDNF in GsMTx4-mediated neuroprotective effects in the ICH model was further supported by the results showing that ANA-12 (BDNF receptor antagonist) abolished the effects of GsMTx4. ANA-12 abolished GsMTx4mediated improvement in behavior, neuroprotection, decrease in neuroinflammation, and oxidative stress, which suggests the critical role of $\mathrm{BDNF}$ in mediating neuroprotective effects of GsMTx4. It is the first report describing that GsMTx4 may exert beneficial effects by increasing the levels of BDNF in the brain. However, there is a need for future studies to identify the possible signaling pathways involved in GsMTx4-mediated increase in BDNF levels in the ICH model.

From the results of this present study, it is difficult to identify which mechanosensitive channel is particularly involved in GsMTx4-mediated protective actions. Moreover, the mechanisms involved in GsMTx4-mediated increase in the BDNF levels cannot be ascertained from the current study. Accordingly, the future experimental studies are required to overcome the limitations of this study.

\section{CONCLUSION}

GsMTx4 exerts neuroprotective effects in intracerebral hemorrhage-associated deleterious effects. The increase in BDNF levels along with a decrease in 
neuroinflammation and oxidative stress may contribute to mediating the neuroprotective effects of GsMTx4.

\section{ACKNOWLEDGEMENT}

The authors are grateful to their Institutes for their support and technical services for conducting research.

\section{CONFLICT OF INTEREST}

There are no competing interests among authors.

\section{ABBREVIATIONS}

BDNF: Brain-Derived Neurotrophic Factor; DCFH-DA: 2',7'-dichlorofluorescin diacetate; ICH: Intracerebral Hemorrhage; ROS: Reactive Oxygen Species; TRPV4: Transient Receptor Potential Vanilloid 4.

\section{REFERENCES}

1. Grysiewicz RA, Thomas K, Pandey DK. Epidemiology of ischemic and hemorrhagic stroke: Incidence, prevalence, mortality and risk factors. Neurol Clin. 2008;26(4):871-95. vii. doi:10.1016/j.ncl.2008.07.003

2. Weimar $\mathrm{C}$, Kleine-Borgmann J. Epidemiology, Prognosis and Prevention of Non-Traumatic Intracerebral Hemorrhage. Curr Pharm Des. 2017;23(15):2193-6. doi:10.2174/1381612822666161027152234

3. Ziai WC, Carhuapoma JR. Intracerebral Hemorrhage. Continuum. 2018;24(6):1603-22. doi:10.1212/CON.0000000000000672

4. Pinho J, Costa AS, Araújo JM, Amorim JM, Ferreira C. Intracerebral hemorrhage outcome: A comprehensive update. J Neurol Sci. 2019;398:54-66. doi:10.1016/j.jns.2019.01.013

5. Chen CJ, Ding D, Ironside N, Buell TJ, Elder LJ, Warren A, et al. Statins for neuroprotection in spontaneous intracerebral hemorrhage. Neurology. 2019;93(24):1056-66. doi:10.1212/WNL.0000000000008627

6. LiQ, Wan J, Lan X, Han X, Wang Z, Wang J. Neuroprotection of brain-permeable iron chelator VK-28 against intracerebral hemorrhage in mice. $\mathrm{J}$ Cereb Blood Flow Metab. 2017;37(9):3110-23. doi:10.1177/0271678X17709186

7. Zhao H, Zhang K, Tang R, Meng H, Zou Y, Wu P, et al. TRPV4 Blockade Preserves the Blood-Brain Barrier by Inhibiting Stress Fiber Formation in a Rat Model of Intracerebral Hemorrhage. Front Mol Neurosci. 2018;11:97. doi:10.3389/fnmol.2018.00097

8. Ohnishi M, Kai T, Shimizu Y, Yano Y, Urabe Y, Tasaka S, et al. Gadolinium causes M1 and M2 microglial apoptosis after intracerebral haemorrhage and exerts acute neuroprotective effects. J Pharm Pharmacol. 2020;72(5):709-18. doi:10.1111/jphp. 13235

9. Bae C, Sachs F, Gottlieb PA. The mechanosensitive ion channel Piezo1 is inhibited by the peptide GsMTx4. Biochemistry. 2011;50(29):6295-300. doi:10.1021/bi200770q

10. Alcaino C, Farrugia G, Beyder A. Mechanosensitive Piezo Channels in the Gastrointestinal Tract. Curr Top Membr. 2017;79:219-44. doi:10.1016/ bs.ctm.2016.11.003

11. Morozumi W, Inagaki S, Iwata $\mathrm{Y}$, Nakamura S, Hara H, et al. Piezo channel plays a part in retinal ganglion cell damage. Exp Eye Res. 2020;191:107900. doi:10.1016/j.exer.2019.107900

12. Qiu Z, Guo J, Kala S, Zhu J, Xian Q, Qiu W, et al. The Mechanosensitive Ion Channel Piezo1 Significantly Mediates in vitro Ultrasonic Stimulation of Neurons. iScience. 2019;21:448-57. doi:10.1016/j.isci.2019.10.037

13. Copp SW, Kim JS, Ruiz-Velasco V, Kaufman MP. The mechano-gated channel inhibitor GsMTx4 reduces the exercise pressor reflex in rats with ligated femoral arteries. Am J Physiol Heart Circ Physiol. 2016;310(9):H1233-41. doi:10.1152/ajpheart.00974.2015
14. Alessandri-Haber N, Dina OA, Chen X, Levine JD. TRPC1 and TRPC6 channels cooperate with TRPV4 to mediate mechanical hyperalgesia and nociceptor sensitization. J Neurosci. 2009;29(19):6217-28. doi: 10.1523/ JNEUROSCI.0893-09.2009.

15. Shvedova M, Anfinogenova Y, Atochina-Vasserman EN, Schepetkin IA, Atochin DN. C-Jun N-Terminal Kinases (JNKs) in Myocardial and Cerebral Ischemia/Reperfusion Injury. Front Pharmacol. 2018;9:715. doi:10.3389/ fphar.2018.00715

16. Velasco-Estevez M, Gadalla KKE, Liñan-Barba N, Cobb S, Dev KK, Sheridan GK. Inhibition of Piezo1 attenuates demyelination in the central nervous system. Glia. 2020;68(2):356-75. doi:10.1002/glia.23722

17. Ward CW, Sachs F, Bush ED, Suchyna TM. GsMTx4-D provides protection to the D2.mdx mouse. Neuromuscul Disord. 2018;28(10):868-77. doi:10.1016/j. nmd.2018.07.005

18. Wang J, Ma Y, Sachs F, Li J, Suchyna TM. GsMTx4-D is a cardioprotectant against myocardial infarction during ischemia and reperfusion. J Mol Cell Cardiol. 2016;98:83-94. doi:10.1016/j.yjmcc.2016.07.005

19. Wang G, Yang $Y$, Wang C, Huang J, Wang X, Liu Y, et al. Exploring the role and mechanisms of diallyl trisulfide and diallyl disulfide in chronic constriction-induced neuropathic pain in rats. Korean J Pain. 2020;33(3):216-25. doi:10.3344/kjp.2020.33.3.216

20. Fu G, Wang H, Cai Y, Zhao H, Fu W. Theaflavin alleviates inflammatory response and brain injury induced by cerebral hemorrhage via inhibiting the nuclear transcription factor kappa $\beta$-related pathway in rats. Drug Des Devel Ther. 2018;12:1609-19. doi:10.2147/DDDT.S164324

21. $\mathrm{Xi} \mathrm{T}$, Jin F, Zhu Y, Wang J, Tang L, Wang Y, et al. MicroRNA-126-3p attenuates blood-brain barrier disruption, cerebral edema and neuronal injury following intracerebral hemorrhage by regulating PIK3R2 and Akt. Biochem Biophys Res Commun. 2017;494(1-2):144-51. doi:10.1016/j.bbrc.2017.10.064

22. Hua Y, Schallert T, Keep RF, Wu J, Hoff JT, Xi G. Behavioral tests after intracerebral hemorrhage in the rat. Stroke. 2002;33(10):2478-84. doi:10.1161/01.str.0000032302.91894.0f

23. Yuan R, Fan H, Cheng S, Gao W, Xu X, Lv S, et al. Silymarin prevents NLRP3 inflammasome activation and protects against intracerebral hemorrhage. Biomed Pharmacother. 2017;93:308-15. doi:10.1016/j.biopha.2017.06.018

24. Romac JMJ, Shahid RA, Swain SM, Vigna SR, Liddle RA. Piezo1 is a mechanically activated ion channel and mediates pressure induced pancreatitis. Nat Commun. 2018;9(1):1715. doi:10.1038/s41467-018-04194-9

25. Han Y, Liu C, Zhang D, Men H, Huo L, Geng Q, et al. Mechanosensitive ion channel Piezo1 promotes prostate cancer development through the activation of the Akt/mTOR pathway and acceleration of cell cycle. Int $\mathrm{J}$ Oncol. 2019;55(3):629-44. doi:10.3892/ijo.2019.4839

26. Roh J, Hwang SM, Lee SH, Lee K, Kim YH, Park CK. Functional Expression of Piezo1 in Dorsal Root Ganglion (DRG) Neurons. Int J Mol Sci. 2020;21(11):3834. doi:10.3390/ijms21113834

27. Beech DJ, Xiao B. Piezo channel mechanisms in health and disease. J Physiol. 2018;596(6):965-7. doi:10.1113/JP274395

28. Lepannetier S, Gualdani R, Tempesta S, Schakman O, Seghers F, Kreis A, et al. Activation of TRPC1 Channel by Metabotropic Glutamate Receptor mGluR5 Modulates Synaptic Plasticity and Spatial Working Memory. Front Cell Neurosci. 2018;12:318. doi: 10.3389/fncel.2018.00318.

29. Chen X, Taylor-Nguyen NN, Riley AM, Herring BP, White FA, Obukhov AG. The TRPC6 inhibitor, larixyl acetate, is effective in protecting against traumatic brain injury-induced systemic endothelial dysfunction. J Neuroinflammation. 2019;16(1):21. doi: 10.1186/s12974-019-1407-6.

30. Cordeiro JL, Neves JD, Nicola F, Vizuete AF, Sanches EF, Gonçalves CA, et al. Arundic Acid (ONO-2506) Attenuates Neuroinflammation and Prevents Motor Impairment in Rats with Intracerebral Hemorrhage. Cell Mol Neurobiol. 2020. Published online September 11, 2020. doi:10.1007/s10571-02000964-6

31. Duan X, Wen Z, Shen H, Shen M, Chen G. Intracerebral Hemorrhage, Oxidative Stress, and Antioxidant Therapy. Oxid Med Cell Longev. 2016. doi:10.1155/2016/1203285

32. Park SP, Kim BM, Koo JY, Cho H, Lee CH, Kim M, et al. A tarantula spider toxin, GsMTx4, reduces mechanical and neuropathic pain. Pain. 2008;137(1):208-17. doi:10.1016/j.pain.2008.02.013 
33. Lopez JR, Kolster J, Uryash A, Estève E, Altamirano F, Adams JA. Dysregulation of Intracellular $\mathrm{Ca}^{2+}$ in Dystrophic Cortical and Hippocampal Neurons. Mol Neurobiol. 2018;55(1):603-18. doi:10.1007/s12035-016-0311-7

34. Guan J, Zhang B, Zhang J, Ding W, Xiao Z, et al. Nerve regeneration and functional recovery by collagen-binding brain-derived neurotrophic factor in an intracerebral hemorrhage model. Tissue Eng Part A. 2015;21(1-2):62-74. doi:10.1089/ten.TEA.2014.0139

35. Miao H, Li R, Han C, Lu X, Zhang H. Minocycline promotes posthemorrhagic neurogenesis via M2 microglia polarization via upregulation of the TrkB/ BDNF pathway in rats. J Neurophysiol. 2018;120(3):1307-17. doi:10.1152/ jn.00234.2018

36. Ko HR, Ahn SY, Chang YS, Hwang I, Yun T, Sung DK, Sung SI, Park WS, Ahn JY. Human UCB-MSCs treatment upon intraventricular hemorrhage contributes to attenuate hippocampal neuron loss and circuit damage through
BDNF-CREB signaling. Stem Cell Res Ther. 2018;9(1):326. doi:10.1186/ s13287-018-1052-5.

37. Jacques-Fricke BT, Seow Y, Gottlieb PA, Sachs F, Gomez TM. Ca ${ }^{2+}$ influx through mechanosensitive channels inhibits neurite outgrowth in opposition to other influx pathways and release from intracellular stores. J Neurosci. 2006;26(21):5656-64. doi: 10.1523/JNEUROSCI.0675-06.2006. Gottlieb PA, Barone T, Sachs F, Plunkett R. Neurite outgrowth from PC12 cells is enhanced by an inhibitor of mechanical channels. Neurosci Lett. 2010;481(2):115-9. doi: 10.1016/j.neulet.2010.06.066.

\section{PICTORIAL ABSTRACT}

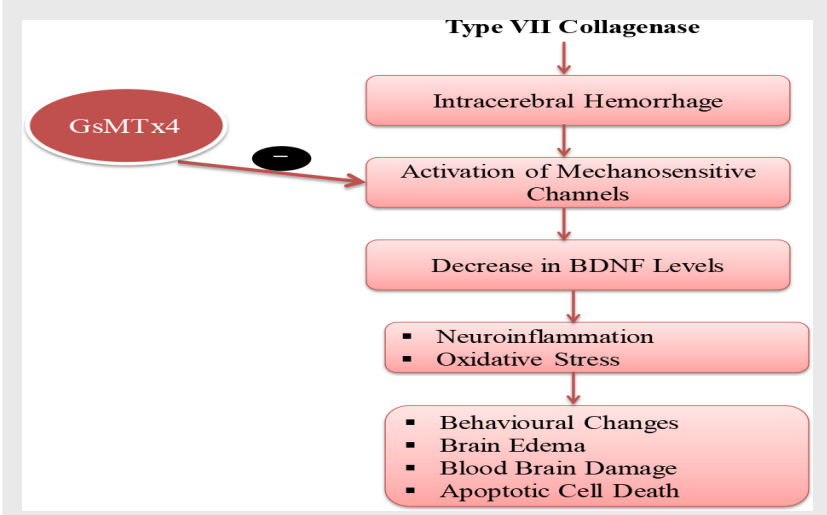

\section{SUMMARY}

This study reported the beneficial effects of selective Piezo 1 channel blocker, GsMTX4 in the intracerebral hemorrhage $(\mathrm{ICH})$ model in rats. GsMTx4 reduced $\mathrm{ICH}$-associated behavioral changes, preserved the blood-brain barrier, prevented brain edema and decreased apoptosis. It also increased the levels of BDNF, decreased neuroinflammation and oxidative stress. The blocker of BDNF, ANA-12, abolished the neuroprotective effects of GsMTx4 suggesting the important role of BDNF. It is concluded that GsMTx4 exerts neuroprotective effects in the $\mathrm{ICH}$ model due to an increase in BDNF, decrease in neuroinflammation, and oxidative stress.

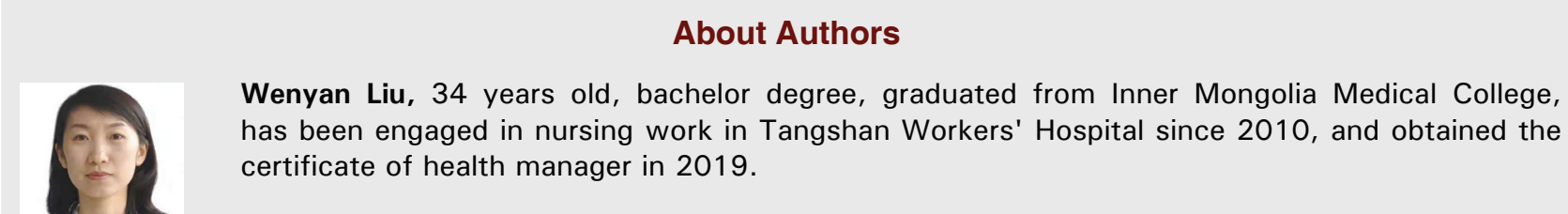
certificate of health manager in 2019.

Guiyu Zhang, 48 years old, bachelor degree, graduated from Chengde Medical College and has been working in Tangshan Workers' Hospital for 25 years.

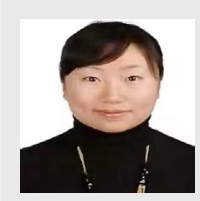

Jin Wang, 37 years old, bachelor degree, graduated from Hebei Union University and has been working in the Second Department of Neurosurgery of Tangshan Workers' Hospital for 15 years.

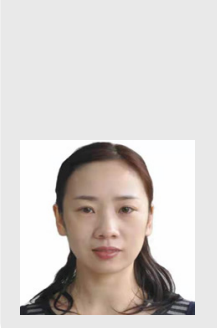

Jing Liu, 43 years old, bachelor degree, graduated from Central South University and has been working in Tangshan Workers' Hospital for 20 years.

Yu Yuan, 35 years old, graduated from Hebei University and has been working in Tangshan Workers' Hospital for 8 years 


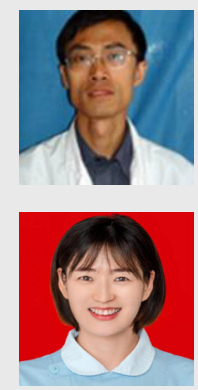

Jun Hong, 53 years old, M.D graduated from Hebei Medical University and has been worked in Tangshan Workers' Hospital 20 years.

Yi Ding, 39 years old, bachelor degree, graduated from Luzhou Medical College. She has been engaged in nursing work at the Central Hospital of Jiangjin District, Chongqing City since 2006, and obtained the title of Deputy Chief Nurse in 2019.

Cite this article: Liu W, Zhang G, Wang J, Liu J, Yuan Y, Hong J, Ding Y. Exploring the Neuroprotective Effects of Mechanosensitive Channel Blocker, GsMTX4 in Intracerebral Hemorrhage Model in Rats. Indian J of Pharmaceutical Education and Research. 2021;55(3):756-64. 